Int. J. Electrochem. Sci., 13 (2018) $1803-1812$

\title{
Structure and Electrochemical Properties of Nitrogen Doped Diamond-like Carbon Film Synthesized by Low Temperature Neutral Beam Enhanced Chemical Vapor Deposition
}

\author{
Qiuhe Wang ${ }^{1}$, Xijiang Chang ${ }^{1,2 *}$, Yoshiyuki Kikuchi ${ }^{2,3}$, Kumi. Y. Inoue ${ }^{4,5}$, Tomohiro Kubota ${ }^{2}$, \\ Tomokazu Matsue, 5,6, Toshihisa Nozawa ${ }^{3}$ and Seiji Samukawa ${ }^{2,6}$ \\ ${ }^{1}$ College of Electronic \& Electric Engineering, Shanghai University of Engineering Science, Shanghai, \\ 201620, China \\ ${ }^{2}$ Institute of Fluid Science, Tohoku University, Sendai, 980-8577, Japan \\ ${ }^{3}$ TEL Technology Center Sendai, Tokyo electron limited, Sendai, 981-3137, Japan \\ ${ }^{4}$ Micro System Integration Center ( $\mu$-SIC), Tohoku University, Sendai, 980-0845, Japan \\ ${ }^{5}$ Graduate School of Environmental Studies, Tohoku University, Sendai, 980-8579, Japan \\ ${ }^{6}$ World Premier International Center Initiative, Advanced Institute for Materials Research, Tohoku \\ University, Sendai, 980-8577, Japan \\ *E-mail: $\underline{091031001 @ \text { fudan.edu.cn }}$
}

doi: $10.20964 / 2018.02 .11$

Received: 27 September 2017 / Accepted: 19 November 2017 / Published: 28 December 2017

We report nitrogen doping diamond-like carbon film synthesis by a new technique called neutral beam enhanced chemical vapor deposition. The deposition is processed in UV/electron damage-free environment, and the substrate temperature is controlled at room temperature. The film structure was investigated by X-ray photoelectron spectroscopy and Fourier transform infrared spectroscopy. The $\mathrm{N} / \mathrm{C}$ ratio is 0.26 , and doped nitrogen atoms exist in forms of amine and inside the aromatic ring, which helps increase film electrical conductivity. Cyclic voltammograms were carried out and the results indicate the DLC: N film qualified as electroanalysis sensor material.

Keywords: DLC film, NBECVD, cyclic voltammograms

\section{FULL TEXT}

(C) 2018 The Authors. Published by ESG (www.electrochemsci.org). This article is an open access article distributed under the terms and conditions of the Creative Commons Attribution license (http://creativecommons.org/licenses/by/4.0/). 